\title{
Mukbang and Disordered Eating: A Netnographic Analysis of Online Eating Broadcasts
}

\author{
Mattias Strand $^{1,2}$ (D) Sanna Aila Gustafsson ${ }^{3}$
}

Published online: 10 April 2020

(C) The Author(s) 2020

\begin{abstract}
Mukbang is a recent Internet phenomenon in which video recordings of hosts eating large amounts of food are streamed on an online video platform. It originated in South Korea around 2014 and has since become a global trend. The aim of this study was to explore how viewers of mukbang videos relate their audience experiences to symptoms of disordered eating. A qualitative analysis of YouTube comments and Reddit posts on the topic of mukbang and disordered eating was performed, employing a netnographic approach. Two overarching themes were identified: a viewer perspective, by which users discuss mukbang without describing any personal involvement, and a participant perspective, by which users describe their own experiences of affects and behaviors in response to watching mukbang. Several topical categories emerged, describing how watching mukbang can both limit and increase eating, reduce loneliness and guilt, and become self-destructive. For some, mukbang appears to be a constructive tool in increasing food intake, preventing binge eating, or reducing loneliness; for others, it is clearly a destructive force that may motivate restrictive eating or trigger a relapse into loss-of-control eating. Notably, watching mukbang is not necessarily experienced as either helpful or destructive, but instead as simultaneously useful and hurtful.
\end{abstract}

Mattias Strand

mattias.strand@ki.se

1 Stockholm Centre for Eating Disorders, Wollmar Yxkullsgatan 27B, 11850 Stockholm, Sweden

2 Centre for Psychiatry Research, Department of Clinical Neuroscience, Karolinska Institutet, \& Stockholm Health Care Services, Stockholm County Council, 17177 Stockholm, Sweden

3 University Health Care Research Center, Faculty of Medicine and Health, Örebro University, 70182 Örebro, Sweden 
Keywords Eating disorders · Disordered eating - Binge eating · Body image · Social media

\section{Introduction: South Korean Eating Broadcasts Gone Global}

The word mukbang (먹방; also stylized as muk-bang or meokbang) is a Korean abbreviation for 'eating show'-a concise summary of this recent Internet phenomenon. A mukbang is a video recording of a host occasionally preparing or arranging but most importantly eating large amounts of food, usually while leisurely chatting with the audience, that is streamed or posted on an online video platform. The amounts of food ingested in a typical mukbang are conspicuously large; sometimes this involves large amounts of a single food-item (such as ramen noodles or fried chicken) but a mukbang may, for example, also consist of multiple doubleportion dishes.

Many South Korean mukbang hosts originally started their broadcasting careers on the Korean live video platform AfreecaTV. However, the mukbang phenomenon has rapidly spread on a global level and a large number of mukbang videos can also be found on internationally oriented platforms such as YouTube, where they are not necessarily live-streamed. According to Google Trends, mukbang started trending as an online search term in South Korea during Winter 2014 and globally in Autumn 2014 (Google Trends 2018).

In some cases, Korean mukbang hosts on AfreecaTV earn money for their 'performance' by viewer contributions in virtual currency; not seldom, hosts can be heard naming and thanking viewers in real time when they notice that someone has contributed money. Reportedly, the most popular mukbang hosts can earn as much as 10,000 United States dollars a month (Quartz 2016; Chu 2018). It can be assumed that some of this income finances further food purchases, such as in mukbangs involving large amounts of sushi or shellfish. However, most foods consumed in mukbang videos appear to be relatively cheap; e.g., multiple packs of instant ramen noodles. Notably, South Korea has in the past years had the world's fastest wireless Internet service and WI-FI hubs are ubiquitous, which has contributed to transforming Korean video streaming from desktop-based to mobile-based and to the further integration of online media into most aspects of life (Quartz 2016). In this context, mukbang has also been described as the most recent tool in promoting Korean cuisine abroad and as a vital part of Hallyu, or the Korean Wave, a term referring to the phenomenal international growth of Korean popular culture encompassing, for example, K-pop music, art house cinema, and television dramas since the 1990s and onwards (Chu 2018).

Mukbang has been understood as originating in "the loneliness of unmarried or uncoupled Koreans, in addition to the inherently social aspects of eating in Korea" (Quartz 2016). In Korean society, dining is a deeply social activity and many Koreans find it almost unthinkable to sit and eat alone, not least in public; hence, mukbang has been described as a virtual substitute for socializing while eating on your own (Jackson 2018). In parallel with this tendency, South Korean health 
officials have expressed great concern with a recent surge in obesity among Koreans and have suggested that phenomena such as mukbang could require more attention and possibly even some form of government regulation (Chu 2018).

The mukbang phenomenon shares some common characteristics with the socalled cheatmeals-i.e., large meals that people that follow a strict diet and/or an intensive physical exercise regime occasionally 'allow' themselves as a reward or an incentive-that have previously been described and analyzed in relation to disordered eating (Pila et al. 2017). Furthermore, many mukbang videos are perceived and labeled by users as 'ASMR', which is short for autonomous sensory meridian response: a sought-after tingling sensation in the crown of the head upon experiencing or watching certain intricate audio-visual triggers such as gentle whispering, the turning of book pages, or somebody slurping and chewing in a delicate manner (Poerio et al. 2018).

Disordered eating is a broad term describing various types of problematic eating behaviors, such as restrictive eating, binge eating, and associated compensatory behaviors (e.g., vomiting, laxative misuse, or excessive physical exercise). Often, but not always, these behaviors stem from body image concerns. Disordered eating may exist on a subclinical level or be a part of an overall clinical picture that fulfills formal diagnostic criteria for an eating disorder, such as anorexia nervosa, bulimia nervosa, binge eating disorder, or avoidant/restrictive food intake disorder (ARFID) (American Psychiatric Association 2013). Anorexia nervosa is characterized by restriction of energy intake leading to a significantly low body weight, intense fear of weight gain, and a disturbed experience of one's own body weight or shape. Bulimia nervosa is characterized by recurrent episodes of binge eating, inappropriate compensatory behaviors to prevent weight gain, and excessive emphasis on weight or shape in self-evaluation. Binge eating disorder is characterized by recurrent episodes of binge eating without compensatory behaviors. ARFID is characterized by restrictive eating due to other factors than body image concerns, such as sensory selectivity, low appetite, or dysphagia. However, subclinical symptoms of disordered eating in the absence of a full eating disorder are significantly more common: during adolescence, around $24 \%$ of girls and $16 \%$ of boys report disordered eating (Hautala et al. 2008).

Anecdotal reports in blog posts and magazines have described how watching mukbang videos can trigger and reinforce disordered eating behaviors such as binge eating or purging behaviors, but that it may actually also help some individuals to restrain from binge eating or encourage overly restrictive eaters to grow an appetite and become more comfortable in eating in a social setting (Kim 2017; Quartz 2016). Hence, mukbang has been described as "a double-edged sword" in regard to disordered eating (Kim 2017): depending on the viewer context, it appears to be both potentially helpful and destructive. However, no formal scientific exploration of the mukbang phenomenon and its relation to disordered eating has been published.

In the current exploratory study, a netnographic qualitative content analytic approach was employed with the aim of understanding how viewers of online mukbang videos relate their audience experiences to symptoms of disordered eating, such as restrictive eating, binge eating, or purging behaviors. Specific questions that 
we wanted to explore were: Are mukbang videos perceived as influencing eating and/or purging behaviors in viewers? If so, in what ways are they described as triggering disordered eating behaviors? In what ways are they, on the contrary, described as potentially helpful for viewers in coping with and recovering from disordered eating?

\section{Methodology}

Netnography was originally developed as a qualitative method for the investigation of consumer behaviors in Internet communities, with an emphasis on the researcheras-participant (Kozinets 1998). Over the years, however, netnography has increasingly been used as a strictly observational approach in health care research, not least for studies on sensitive topics where it may be difficult to negotiate access or recruit informants for a more traditional qualitative design (Langer and Beckman 2005). A netnographic approach has been used in, for example, research on pro-anorexia communities (Dyke 2013) and online peer support in diabetes mellitus (Tenderich et al. 2019). The terms netnography, digital ethnography, and digital anthropology are sometimes used interchangeably. However, although the practical methodology may not differ very much, a key conceptual difference is that in a netnographic analysis, the online community under study is seen as a habitat in its own right, governed by sociocultural codes that are essentially separate from 'offline' life in many respects. In contrast, the digital ethnographer generally approaches an online community merely as an extension of or a complement to offline ethnographic studies. We do not wish to overstate these differences; even so, we believe that a number of unique features of mukbang are intimately connected to the online nature of the phenomenon and that a netnographic approach is therefore preferable.

In the present study, two modes of inquiry were employed. First, viewer comments of popular mukbang videos on YouTube were analyzed. YouTube (youtube.com) is a video-sharing platform that allows its users to upload, view, rate and comment on videos, most of which are user-generated. The wide variety of available content includes video blogs, live streams, music videos, short and documentary films, television clips, movie trailers, and educational videos. As of February 2019, YouTube is rated as the 2nd most visited Web site globally by Alexa Internet, Inc. (Alexa Internet 2019). Similar analyses of YouTube comments have previously been utilized in studies on topics such as non-suicidal self-injury (Lewis et al. 2012), weight stigma (Jeon et al. 2018), and pro-anorexia content (Oksanen et al. 2015).

On YouTube, the three most viewed videos from five popular mukbang hosts- $\mathrm{i}$. e., a total of 15 videos (see Table 1)-were included in this analysis. These particular hosts were chosen mainly based on their popularity in terms of number of viewers, but also so as to reflect the geographical diversity of the mukbang phenomenon: as seen in Table 1, three of the hosts are based in South Korea, one in Japan, and one in the United States. Data on the basic characteristics of mukbang content in the 15 included videos are provided in Table 1. With the help of a free online tool (ytcomments.klostermann.ca) for downloading YouTube viewer 


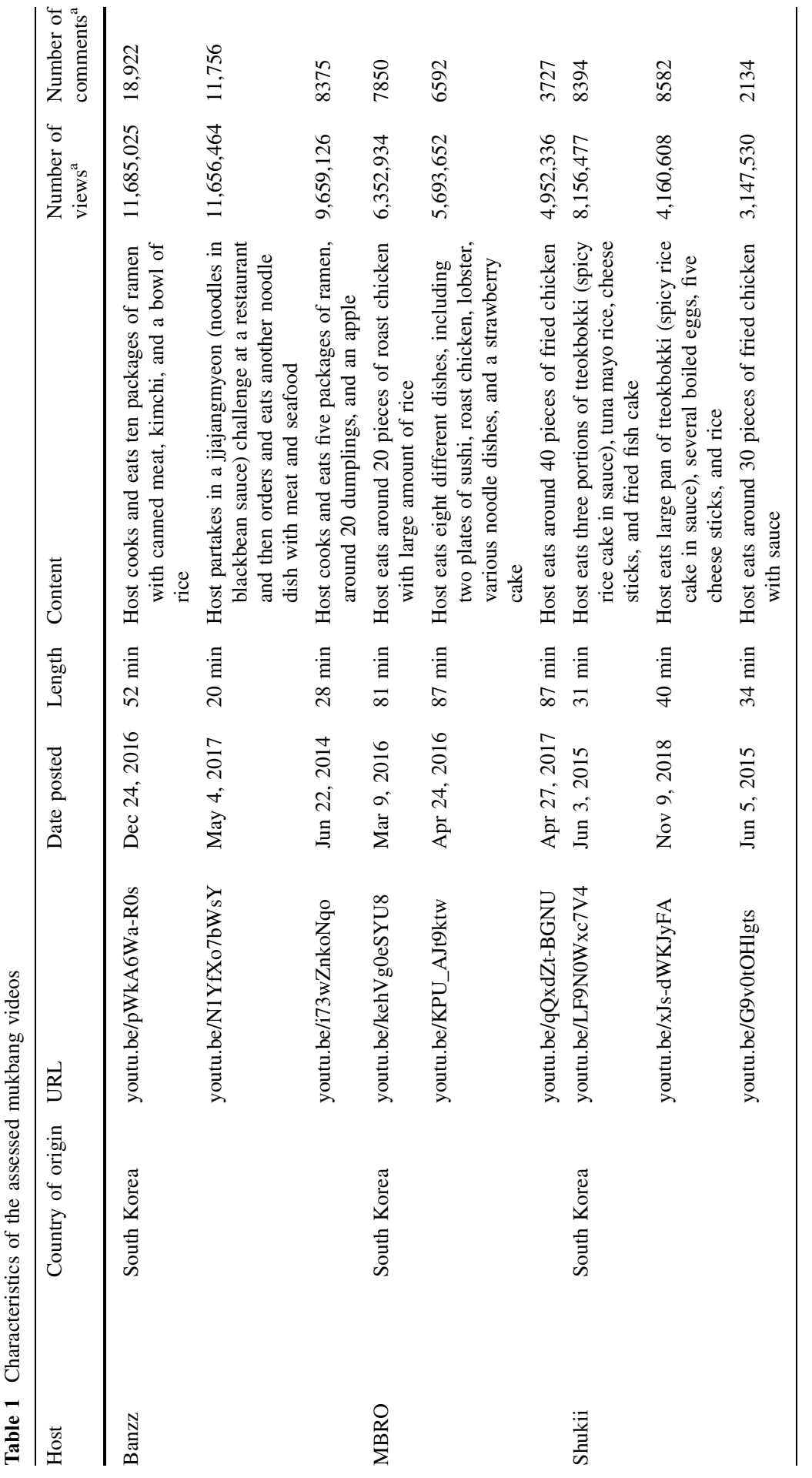




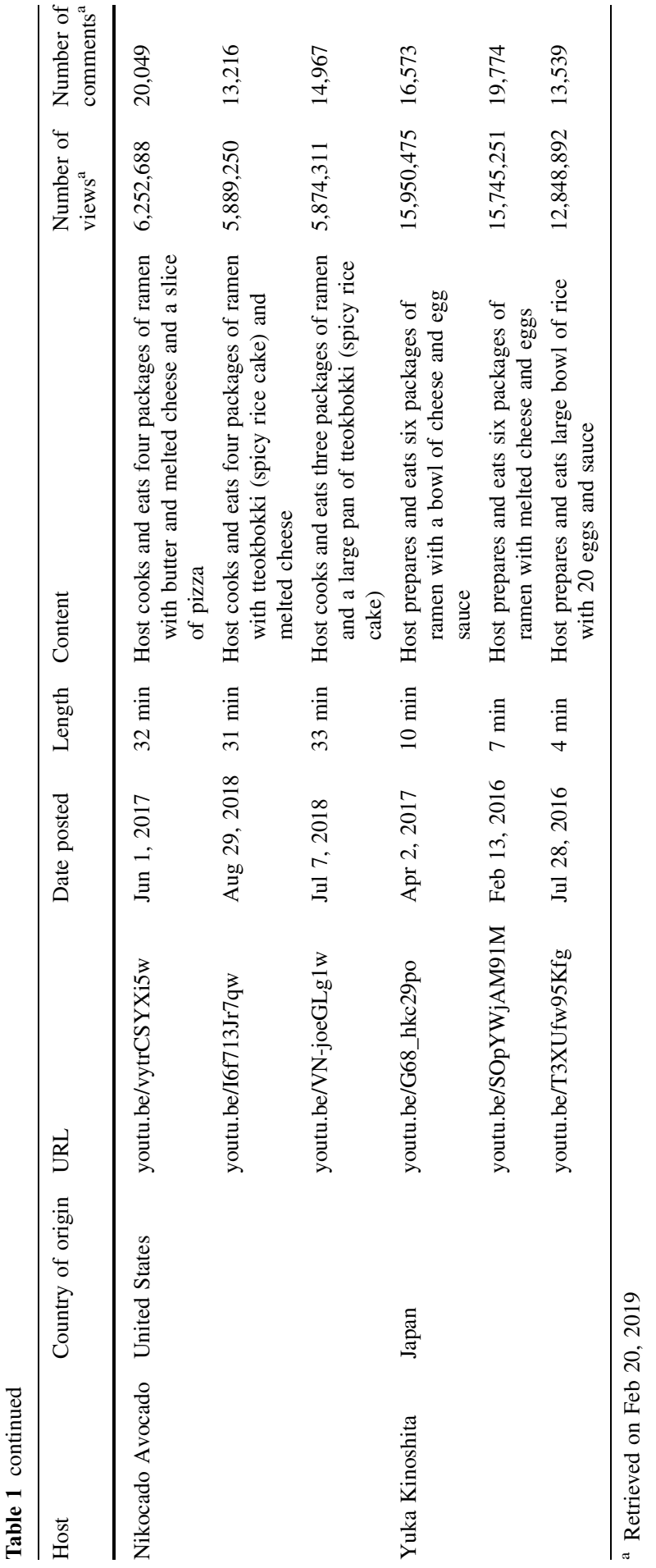


comments in JavaScript Object Notation (.json) format, which is compatible with Microsoft Excel, the full comments section of these videos was retrieved on Feb 20, 2019 and then examined in close detail in search of viewer comments on the topics of disordered eating and body image. Here, an English-only language limit was applied. Naturally, this limits the transferability of the findings to other language contexts and we elaborate on the implications of this in the Discussion section. In total, over 174,000 YouTube comments were assessed. At this stage, any user comments relating to the research questions outlined above were included; thus, comments discussing symptoms of disordered eating (such as restrictive eating, binge eating, or purging behaviors), body image concerns, as well as the sociocultural role of mukbang were extracted for closer examination. In contrast, the large number of comments expressing amazement (e.g., "omfg yo"), admiration (e.g., "I love youuuu <3"), or disgust (e.g., "Ewww ur grossing me out") in a more general sense about the amounts of food eaten in the broadcasts were not included in the final qualitative analysis. As an illustration of the volume of comments excluded at this stage, around 200 variants of "I love cheese!", several hundreds of "Hi!" and "Yummy!", and well over 1000 versions of "R u gay???" were removed. These types of brief, nonsensical, or pejorative comments made up the bulk of the data.

Second, online posts on the topic of mukbang and disordered eating on Reddit were analyzed. Reddit (reddit.com) is a user-generated news and discussion Web site consisting of free-access topical or demographical discussion communities known as 'subreddits'. As of February 2019, Reddit claims to have over 330 million monthly active users and is rated as the 17th most visited Web site globally by Alexa Internet, Inc., with $53.4 \%$ of its visitors originating in the United States making it the most popular English-language discussion community (Alexa Internet 2019). As a complement to the YouTube data, Reddit was chosen as an additional data source specifically because of its discussion forum format, which hypothetically encourages somewhat longer, more self-disclosing, and reciprocal comments. Reddit content has previously been analyzed in studies on various topics, such as suicidology (Aladağ et al. 2018), cannabis use (Sowles et al. 2017), pro-eating disorders communities (Sowles et al. 2018), and gout (Derksen et al. 2017).

For this study, fifteen relevant subreddits that contained discussions about mukbang were identified, utilizing the Reddit search function: r/1200isplenty, r/AnorexiaNervosa, r/BingeEatingDisorder, r/bulimia, r/confession, r/EDAnonymous, r/fasting, r/fatlogic, r/fatpeoplestories, r/food, r/fuckeatingdisorders, r/gainit, $\mathrm{r} /$ korea, $\mathrm{r} /$ loseit, and r/nutrition. In total, these subreddits had more than 19 million subscribers when data were retrieved on Feb 20, 2019. Data on user experiences of watching mukbang were collected by identifying specific posts or threads related to this subtopic with the help of the Reddit search function. Similar inclusion and exclusion criteria as in the analysis of YouTube comments were used. No time limits were applied, i.e., all comments available up to the date of data retrieval were included in the analysis.

It should be noted that both YouTube and Reddit allow users to respond to other users' previous comments. In the data retrieved from Reddit, we could easily follow such exchanges. In the data retrieved from YouTube, however, this was not possible due to the fact that the.json data format did not allow for a hierarchical display of 
comments and subcomments. Even so, the context of the comments often made it possible to distinguish when a user responded to another user's comment, as seen in the interactions described in the Results section.

The first pre-analysis phase of the assessment-i.e., identifying relevant YouTube videos and subreddits, retrieving comment data, and scanning these data for irrelevant or redundant information-was performed by the first author alone, due to the sheer number of comments and the largely mechanical nature of the work. In total, 1316 user comments (986 YouTube user comments and 330 Reddit user comments) were included in the final qualitative content analysis. The collected data were then analyzed using the qualitative analysis software NVivo 11. Qualitative content analysis is usually the preferred method of data analysis in netnographic studies (Langer and Beckman 2005); here, a directed approach to qualitative content analysis (Hsieh and Shannon 2005) was applied, guided by the research questions outlined above. Thus, data were primarily analyzed in a 'top-down' manner whereby any data relevant to our research questions were coded. When appropriate, a single comment expressing multiple or ambivalent statements could receive more than one coding label. In a second step, all relevant data were categorized in an iterative 'bottom-up' approach by the first author. Next, the second author independently coded all data in accordance with the preliminary coding scheme, in order to validate the categories. This step leads to some minor changes to the coding scheme, resulting in two overarching themes and 11 categories. An interrater reliability above $90 \%$ was achieved and any interrater disagreement was resolved by consensus.

The study was preregistered on the Open Science Framework (osf.io/4w32d). Since the study was of a strictly observational nature utilizing publically available online data, no ethical vetting was necessary according to Swedish law. In order to ensure full anonymity, no online user names (anonymous or otherwise) were collected, analyzed, or presented. For a more detailed discussion on research ethics in netnographic studies, see Langer and Beckman (2005).

\section{'Outsider' and 'Insider' Perspectives}

Two overarching themes, each consisting of several categories, were identified among the user comments. The first theme, which we have called the viewer perspective, consisted of 1022 comments that are made from an 'outsider' perspective (see Table 2). Here, users comment and discuss aspects of mukbang without describing any personal involvement. In contrast, in the second theme, which we have called the participant perspective, users take an 'insider' perspective in describing their own experiences of affect and behavior in response to watching mukbang videos (see Table 3). This second theme consisted of 269 comments, notably fewer than the first theme. Since all data were anonymized prior to analysis, we were not able to conclude whether individual users made multiple comments that fitted both of these overarching themes. 


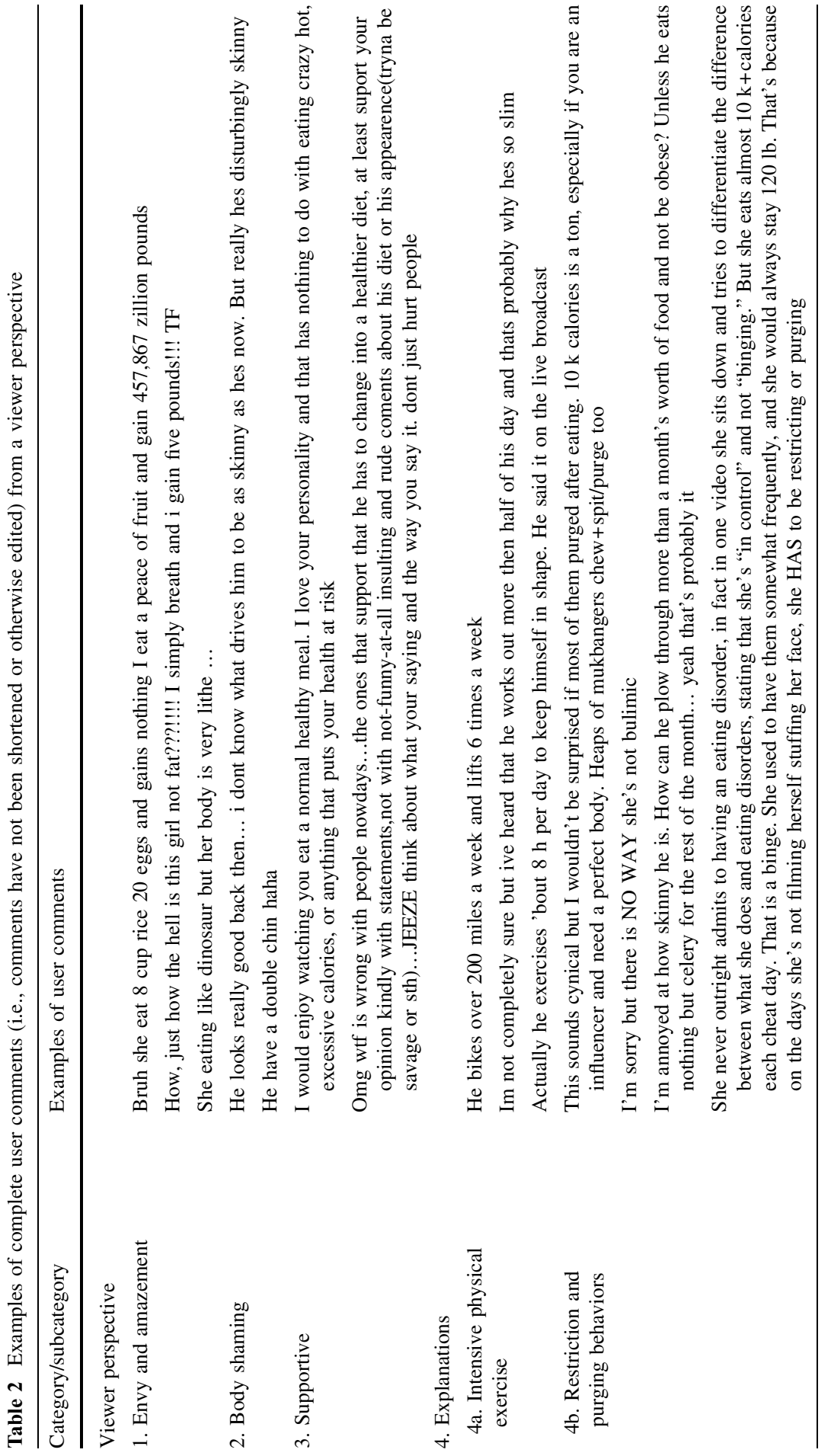




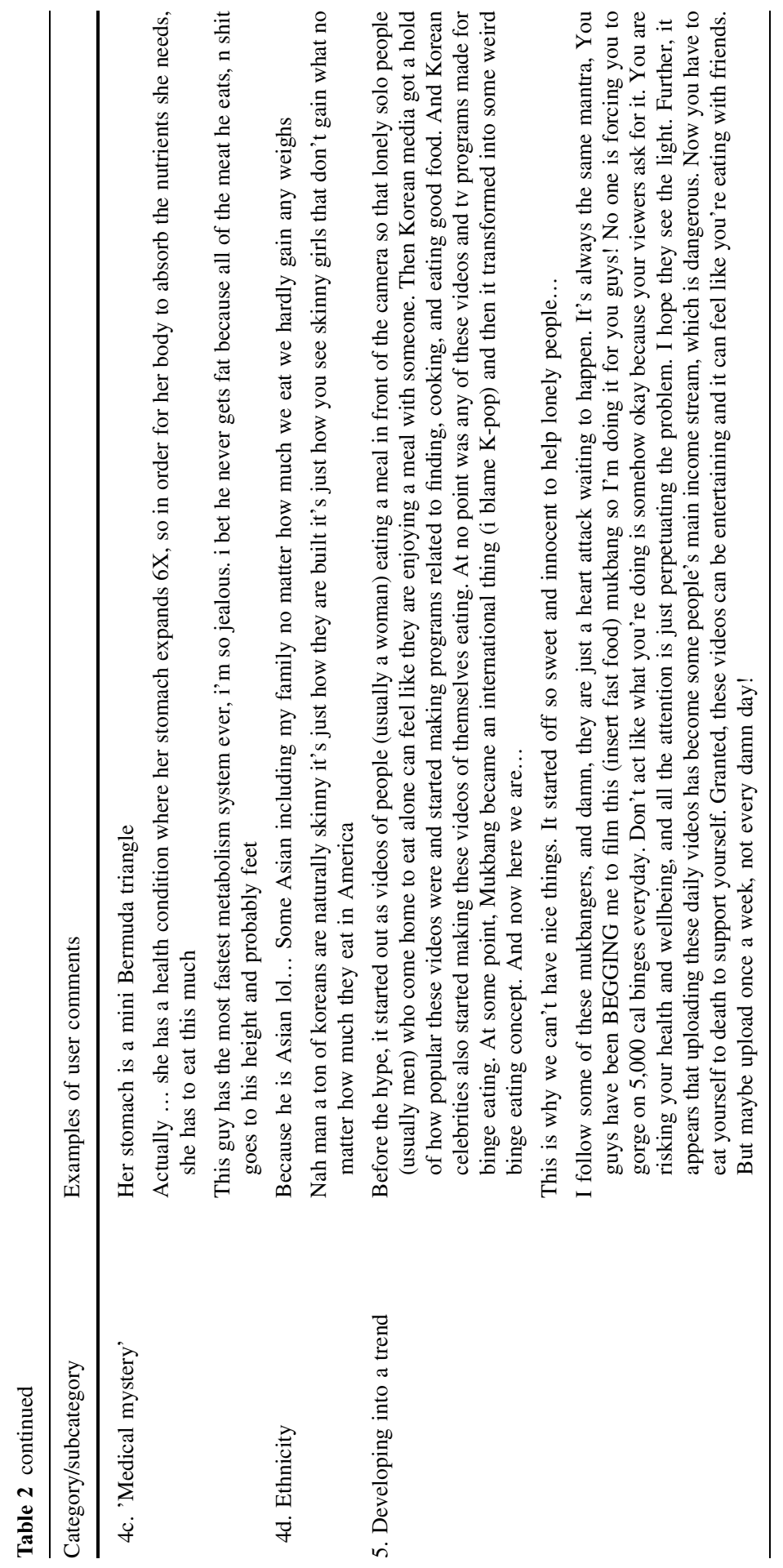




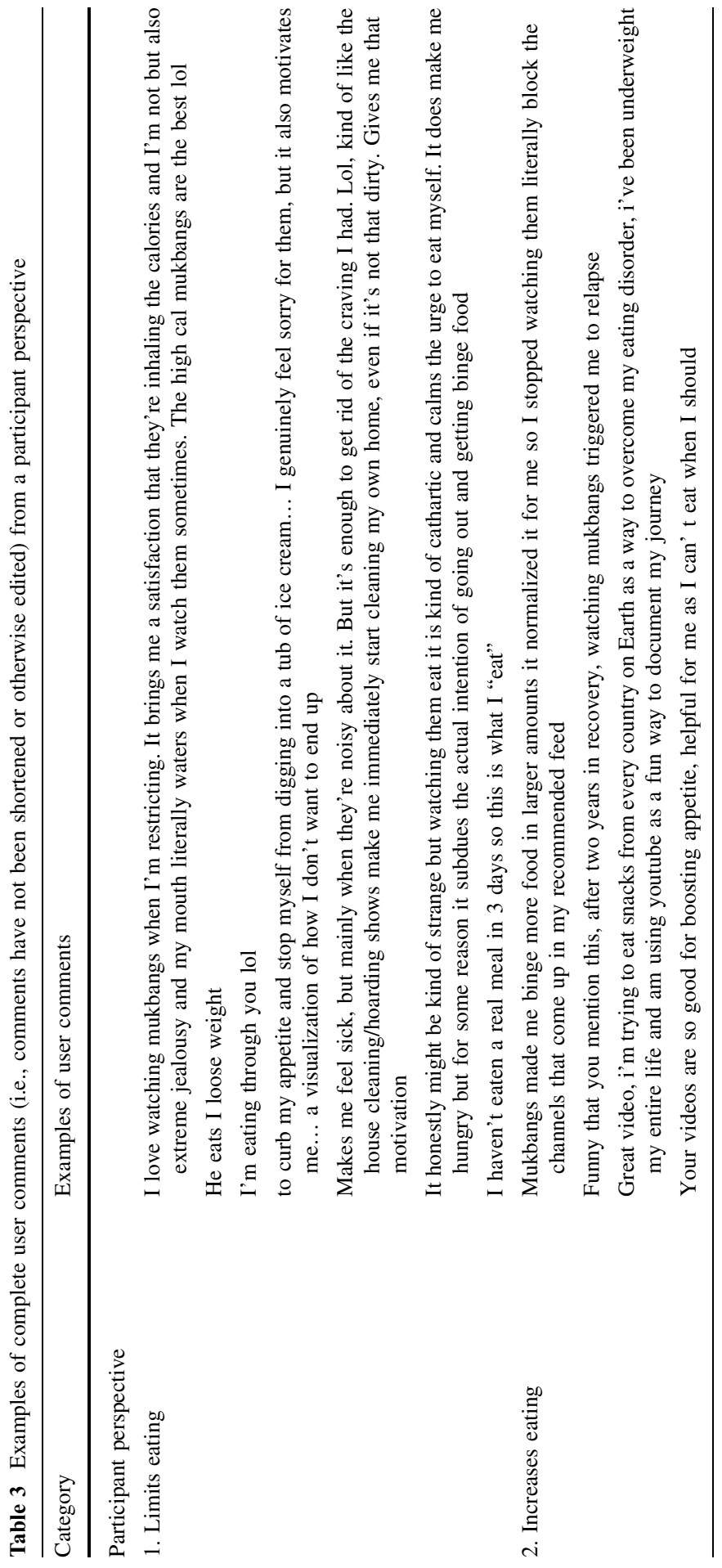




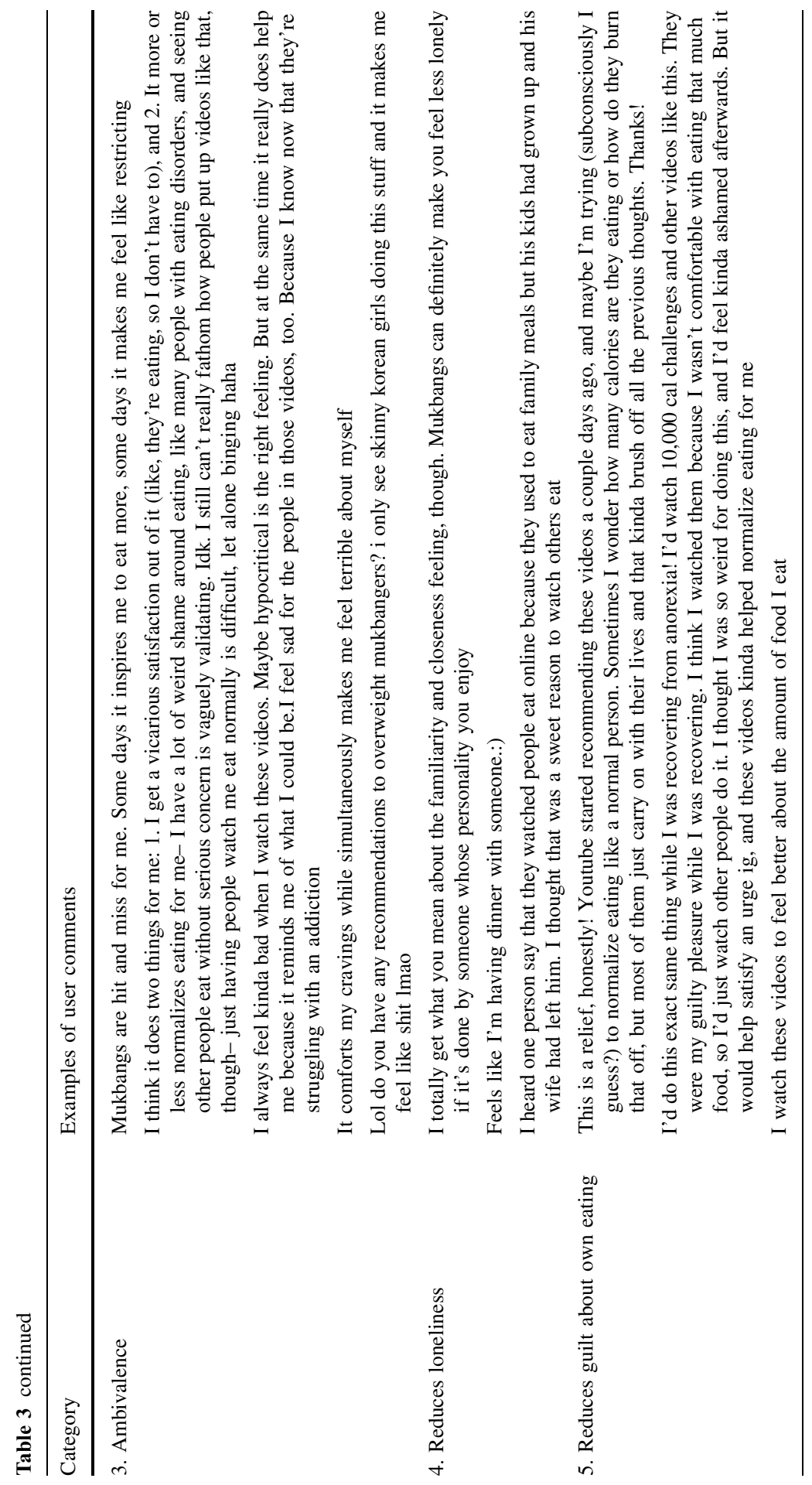




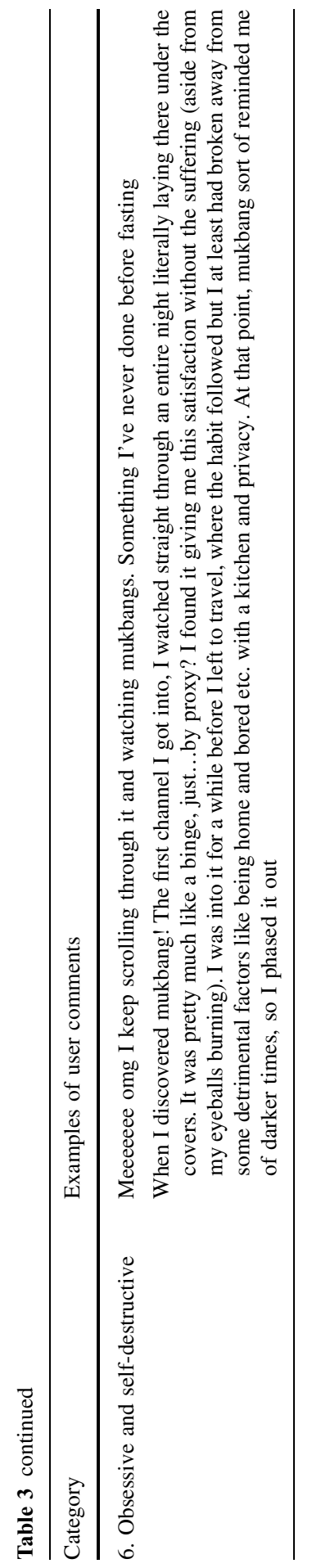




\section{The Viewer Perspective}

Around 400 user comments describe envy and amazement over how mukbang hosts can eat such large amounts of food without apparent weight gain (approximately 400 comments; see Table 2, category 1). Many also express that if they would try the same, they would immediately gain weight in an undesirable way. A gender pattern can be observed in that most of these comments are directed at the two female hosts included in the assessment; however, because of the limited number of mukbang hosts included in the analysis, we did not attempt to perform any formal statistical analyses regarding quantitative comment patterns.

There are also a body shaming comments (approximately 200 comments; see Table 2, category 2). A few of these comments are made on the grounds of mukbang hosts being perceived as "too skinny"; however, a large majority of the body shaming comments are directed at the one host in our assessment who viewers mainly perceived as overweight and thus consisted in fat shaming. Still, there are a number of examples of users expressing a supportive view, calling out other comments as pejorative (approximately 20 comments; see Table 2, category 3). These supportive comments also often encourage mukbang hosts not to feel obligated to challenge themselves or try to impress viewers with excessive amounts of food, but to instead post videos of themselves eating more balanced meals for the sake of their own health.

Many users engage in more or less realistic explanations about how mukbang hosts are able to repeatedly ingest large amounts of food and seemingly not gain weight (approximately 270 comments). Here, several subcategories can be identified. Intensive physical exercise is mentioned as an explanation in a large number of comments (see Table 2, category 4a), although this topic is a subject of debate among viewers. Often, viewers point to the fact that some mukbang hosts themselves explicitly claim to spend several hours a day working out in order to be able to eat large amounts without gaining weight. However, some viewers question this assumption on the grounds that the hosts do not necessarily look very muscular or athletic. Instead, restricting and purging behaviors before and/or after the recording of mukbang videos are brought up as a more realistic explanation, albeit one that viewers assume that mukbang hosts would be more reluctant to admit (see Table 2, category $4 b$ ).

Yet another popular explanation among viewers is that mukbang hosts do not gain weight due to some type of 'medical mystery' (see Table 2, category 4c). Here, some users speculate about somewhat plausible explanations such as hypermetabolism or food passing undigested through the gastrointestinal system. Others, however, engage in highly unrealistic hypothesizing about unnamed rare medical conditions in which a person's stomach is said to expand to the extreme to allow for the ingestion of excessive amounts of food (the numbers six or even 66 times that of a normal-sized ventricle is frequently mentioned, apparently inspired by a pseudomedical television piece aired on a Japanese eating show). Another recurrent version of the medical explanation is that of East Asian ethnicity being associated with fast metabolism and a disposition toward a small body frame size, although this 
explanation is often dismissed as highly anecdotal by other viewers (see Table 2, category 4d).

Another viewer perspective category is that of how the mukbang phenomenon has successively been degenerating into a trend (approximately 30 comments; see Table 2, category 5). Here, commentators frequently explain the idea of mukbang having originated as a live substitute for the immensely important Korean family dinner, targeted at the increasing number of individuals in single-person households working long hours or being shut in their homes gaming or gambling online: "These people need to eat too! But they've been raised to feel absolutely uncomfortable eating by themselves, especially alone at home. So who steps in to fill this most unlikely of markets? Mukbang broadcasters of course!" A recurrent trope is that this benevolent online social eating community has subsequently been appropriated by sensationalist media and metamorphosed into a grotesque 'voyeurist' trend disconnected from its original social context. Whether or not this is an accurate depiction is beyond the scope of this article, but it provides a useful background for the participant perspectives described below.

\section{The Participant Perspective}

Many users describe how watching mukbang makes them limit their own eating (approximately 150 comments; see Table 3, category 1). Individuals who appear to be restricting their eating in an unhealthy way recurrently attest to "eating vicariously" through the mukbang host, i.e., watching mukbang makes them feel that they themselves do not need to eat. Some users engaging in restrictive eating explain this in terms of being repelled by the large amounts of food ingested in the mukbang videos, thereby losing their appetite. A more common experiential description, however, is that of eating 'by proxy' and actually enjoying watching the mukbang host, all the while feeling proud about oneself abstaining from eating. The same overall pattern is found in comments made by users who appear to have a history of binge eating: they sometimes comment on how watching mukbang helps them stave off a binge by finding the amounts of food ingested in the videos repellent, but more often they too describe a more pleasant experience of calm in seeing someone else eat "for" them.

Others describe how watching mukbang increases their own eating (approximately 75 comments; see Table 3, category 2). Here, a majority of users appear to have a history of binge eating and attest to how watching mukbang videos may trigger them to relapse into loss-of-control eating. In contrast, a substantial minority of comments in this category appears to be made in a context of struggling with low appetite and/or selective eating and finding mukbang helpful as an inspiration in increasing food intake. Furthermore, many comments express ambivalence from a participant perspective toward the mukbang phenomenon (approximately 65 comments; see Table 3, category 3). For example, users mention that their response to watching mukbang varies, so that it sometimes leads to increased eating and at other times enforces restriction. Others describe how mukbang evokes several types of affects and behavioral impulses at once, e.g., being inspired to eat less in the moment while simultaneously reducing shame around eating in general, or that they 
enjoy the eating part of the mukbang concept but feel distressed about not being as thin as the mukbang host.

A number of comments relate to the idea of mukbang as being primarily a social phenomenon discussed above. Here, users describe how watching and participating in mukbang reduces loneliness and makes them feel like they are having dinner with someone (approximately 30 comments; see Table 3, category 4). Indeed, some individuals appear to develop a close (albeit one-way) online relationship to a certain mukbang host over time, praising their good-humored personality as a crucial factor in engaging in the mukbang eating community. These comments are sometimes made in conjunction with descriptions of how watching mukbang increases eating. Furthermore, users describe how mukbang reduces guilt about their own eating and that watching mukbang videos is helpful in normalizing eating (e.g., demonstrating that eating can be a joyful experience and that occasional overeating does not automatically need to evoke feelings of guilt; approximately 35 comments; see Table 3, category 5).

Finally, a number of comments mention that watching mukbang can become obsessive and self-destructive (approximately 65 comments; see Table 3, category 6 ). This is sometimes expressed primarily in the form of an excessive fascination with the peculiar conspicuousness of the mukbang concept, but also potentially as part of a pattern of disordered eating behaviors that reinforce the need to keep watching mukbang in order to maintain a restrictive diet. For some, it appears that mukbang can become a destructive way of reducing feelings of boredom.

\section{Discussion: Parasocial Interaction, Modernity, and Ambivalence}

In conclusion, this study shows that users engage with online mukbang videos both as 'outsider' viewers and as 'insider' participants. In some instances, users discuss behaviors that are evidently part of a broader pattern of disordered eating. For example, they describe how they find mukbang useful in keeping a clearly restrictive diet or how watching mukbang may trigger a relapse into binge eating. However, there are also many examples of user comments that describe engaging in mukbang as a way of trying to increase food intake in a context of low appetite or selective eating, or as a way of actually preventing a binge eating episode.

The comments made from a participant perspective were often difficult to categorize in terms of 'healthy' or 'unhealthy'. A comment about how watching mukbang makes the viewer eat more may point to mukbang as a helpful tool for an individual who is struggling with trying to increase the amounts and variety of food accepted and to gain weight. In contrast, the very same comment may be seen as a sign of the potential triggering and unhelpful nature of mukbang if made by an individual with a previous binge eating disorder who has now relapsed into loss-ofcontrol eating. In some instances, the context in which a comment is made makes it possible to determine whether watching mukbang is helpful or unhelpful. More often, however, the context is opaque or ambiguous. We therefore chose to categorize comments made from a participant perspective in terms of whether watching mukbang results in the user limiting or increasing food intake, etc. As seen 
in Table 3, the examples of user comments vary in their description of contextual factors.

Notably, there is often a striking ambivalence in user comments on mukbang. The phenomenon is not necessarily seen as helpful or destructive, but instead simultaneously useful and hurtful. For some, it may evoke several types of affects and behavioral impulses at once, such as limiting food intake in the moment while simultaneously reducing shame around eating in general or experiencing a reduction in loneliness but also distress about the thin body ideal that is often promoted in the videos.

The present findings point to the explicitly social nature of mukbang. This sociality is probably best described in terms of so-called parasocial interaction. The concept of parasocial interaction was originally established in the 1950s to describe how viewers and audiences gradually develop a personal, albeit one-way, relationship to television celebrities, movie stars, and other public figures such as politicians (Horton and Wahl 1956). Subsequently, models of parasocial interaction have been applied in the analysis of a wide variety of mass media phenomena, from children's identification with fictional television characters (Hoffner 1996) to charismatic relationships with televangelists (Diekema 1991). With the emergence of social media in the last decades, there has been a renewed interest in the concept. Indeed, a study commissioned by Google has shown that a majority of young people identify even closer with YouTube creators and video blog hosts than with traditional celebrities (O'Neil-Hart and Blumenstein 2016). Parasocial interaction develops over time as repeated 'meetings' with a performer make viewers experience increasing feelings of friendship and intimacy, much in the same way as they know and understand their flesh-and-blood friends (Ballantine and Martin 2005). Thus, viewing episodes may become an important part of the daily social life of the viewer. Not least, since online social media platforms often allow people to respond to celebrities' messages, the relationship may come to be experienced as even more 'real' or intimate, even though it is (with very rare exceptions) nonreciprocal (Kim and Song 2016). This is probably even further exacerbated by the tendency of many online celebrities to self-disclose and to share everyday life experiences (which may, of course, include food and eating). In the case of mukbang, the non-reciprocity between host and audience may actually be less evident, given that viewers may sometimes donate money in virtual currency in real-time, whereupon they are usually named and thanked directly by the hosts.

There has been some debate about whether or not parasocial interaction should be viewed as a dysfunctional behavior-e.g., as a substitute for non-virtual interpersonal relationships. Some evidence suggests that parasocial interaction may help broaden the scope of an individual's interpersonal relationships, rather than compensate for a lack of closeness (Ballantine and Martin 2005). The findings from the present study indicate that mukbang viewers may indeed develop parasocial relationships with mukbang hosts. Moreover, it seems that they often, if not always, experience these relationships as helpful in reducing loneliness or alleviating feelings of guilt. In the case of body image concerns and thin-ideal internalization, upward social comparison-such as the typical parasocial relation between fan and celebrity - has been shown to be a mediating factor (Fitzsimmons-Craft et al. 2014). 
However, research findings on parasocial interaction and body image are equivocal. One study showed that young women with a desire to be and look like a popular female television character do display more body image concerns. On the other hand, the actual level of parasocial interaction with that character is not associated with body shame or body surveillance (Greenwood 2009). Likewise, another study showed that men who are exposed to images of muscular superheroes tend to feel bad about their own bodies. However, those men that have a strong parasocial relation to said superhero (e.g., as a result of having spent a childhood immersed in comic books) do not display the same tendency and may actually feel better about themselves upon exposure (Young et al. 2013).

Another crucial aspect of mukbang is, undoubtedly, the massive amounts of food consumed. Here, the vital role of excess and exaggeration in the construction of a notion of 'modern life' has often been noted. For example, the concept of conspicuous consumption, introduced by sociologist Thorstein Veblen in the 1920s to explain the emergence of a tendency to uphold social status by display of consumption patterns and leisure time (Veblen 2009), could potentially be evoked to shed light on the mukbang performance. The gendered aspects of modernity, excess, and 'the grotesque' have also been emphasized (Russo 1994). Notably, many twentieth century theorists have tended to describe these phenomena in more or less dystopian terms: as commodity fetishism within a 'society of spectacle' (Debord 1992), as virtuality and hyperreality devoid of meaning (Baudrillard 1983), or as unavoidable aspects of a new media ecology (McLuhan 1964). A straightforward illustration of this pessimistic and cynical tendency is the 1973 French-Italian movie La Grande Bouffe, directed by Marco Ferreri. In this satire on consumerism and bourgeoisie decadence, a group of middle-aged male friends retreats to a villa over the weekend with the purpose of eating themselves to deathanticipating, perhaps, the excesses of twenty-first century mukbang.

A notable exception to this dystopian view of overconsumption and overeating is literary theorist Mikhail Bakhtin's work on the medieval world of François Rabelais. Here, Bakhtin shows how the satirical yet joyous and affirmative depictions of carnivalesque banquets in Rabelais' writing "differ sharply from the images of private eating or private gluttony and drunkenness in early bourgeois literature" (Bakhtin 1984, p. 302). A direct observation is that the mukbang performances included in the present study, weirdness and grotesquerie notwithstanding, have nothing overtly cynical about them. In all 15 assessed mukbang videos, the tone is friendly, intimate, and youthful. As described above, this parasocial quality may not be truly reciprocal and the mukbang phenomenon as such could certainly be critiqued from an anti-consumerist point of view. Even so, the actual performance of mukbang eating is uniformly depicted as the host taking on and joyously conquering the enormous amounts of food rather than the food-ascommodity triumphing over the consumer.

A more accurate comparison, then, may be so-called food porn; i.e., carefully staged images of appealing dishes that are consumed visually on blogs or in glossy magazines rather than viscerally at the dining table. The consumption of food porn has been described as "ingestion without incorporation" (Lavis 2015, p. 201) -an elusive confusion of virtual and actual eating. This very quality may contribute to 
the fascination with food porn, cook books, and recipes that individuals with anorexia nervosa often display as a way of constantly engaging with food without actually eating it (in the everyday meaning of the word). Just as food porn shared on blogs or social media may come to function primarily as a social relationship between people rather than as simply a collection of alluring images (Lavis 2015), it should be clear from the findings of the present study that the mukbang phenomenon is more than just a display of someone eating large amounts of food. Indeed, for some viewers with disordered eating, mukbang appears to fill the very same (para)social function of 'ingestion without incorporation' as do food porn.

This ambiguous stance toward mukbang, seen in many of the analyzed comments, mirrors a broader ambivalent tendency in disordered eating and body dissatisfaction. It has often been noted that ambiguity is at the very core of an eating disorder such as anorexia nervosa: "[Patients] experienced pleasure and disgust, were empowered and disempowered, felt safe yet constantly threatened, were both pure and dirty, and when sickest felt at their best. Anorexia was a constant process of becoming and unbecoming, of having a life by moving toward death." (Warin 2010 , p. 4) Not least, feeling in control while also being controlled by the illness is a very common theme in patient interviews: "It takes control of you, but it can also feel very safe. It's a very confusing illness, because at the moment it's probably got a lot of control over me, in certain ways, and I just want to get away from it, I'm just sick and tired and I'm exhausted, but then it kind of protects you as well, I think, from coping with other things." (Tan et al. 2003, pp. 632-633) This ambivalence is often depicted as two opposing forces involved in an inner struggle for command over the recovery process (see for example Gorse et al. 2013). It may also involve equivocal feelings about the 'food as medicine' paradigm and psychiatric medication (Lester 2014), based on ideas that restrictive eating, for instance, is actually a morally virtuous forms of self-care rather than an alarming symptom (Musolino et al. 2015). However, others have described an ambiguity of a more profound nature in eating disorder narratives, involving breaks with self-continuity and linear recovery trajectories in favor of circular timelines, uncertainty in terms of selves and self-mastery, and irony (Shohet 2007, 2017).

\section{From 'Sweet and Innocent' to Carnivalesque}

As seen in Table 1, the content of the analyzed mukbang videos in terms of the food eaten was surprisingly homogenous. A substantial majority of the broadcasts involved eating large amount of ramen noodles, regardless of geographical origin (South Korea, Japan, or the United States). Although this is certainly not always the case, there appears to be a fascination among viewers with the supposedly Asian features of mukbang. In our analysis, we found a recurrent lamentation about the "sweet and innocent" Korean origin of mukbang (i.e., social eating online targeted at single households) that has subsequently transformed into "an international thing" and, consequently, "some weird binge eating concept" (see Table 2, category 5). Even though further exploration of the successive development of the mukbang phenomenon is beyond the scope of this article, it can be noted that the prevalence 
of disordered eating and body dissatisfaction has been shown to be relatively high in a South Korean context (Jackson, Keel, Lee 2006; Jung and Forbes 2007; Pike and Dunne 2015).

From a 'technical' point of view, mukbang can be described as a pre-planned binge eating episode staged and performed for a virtual audience (sometimes also with a monetizing purpose). Indeed, the explicit language of 'bingeing' is often used in viewer comments and would appear to be a vital factor in the alluring appeal of the phenomenon. Here, comparisons can be made with 'competitive eating' or 'speed eating' shows, where participants compete in eating as much as possible in front of an audience and a series of judges during a specified, usually brief, period of time. Interestingly, such eating contests have been described as combining the spectacle of the medieval carnival with modern ideas of consumerism and abundance (Johnson 2011). For example, the annual Nathan's Hot Dog Eating Contest held in Coney Island, New York City, has been aired on live television to millions of viewers since the early 2000s. Likewise, it has been recognized that popular descriptions of eating disorders often display an ambivalent and uncanny fascination with the 'carnivalesque' image of thinness and illness (Warin 2004). Such connotations may deserve more attention from the research community in the context of online video broadcasting.

To the best of our knowledge, this is the first published study of the mukbang phenomenon from a perspective of disordered eating behaviors and body image. A very large number of YouTube comments and Reddit posts were analyzed during the assessment phase and a high interrater reliability in terms of the identified thematic categories was achieved. Still, the exploratory nature of the study and the small-scale scope of the included video material (i.e., 15 broadcasts from a total of five mukbang hosts) limit the transferability of the findings. For example, although the overall impression during coding was that saturation was reached in the data, we cannot exclude the possibility that including other mukbang hosts and/or videos in the analysis would yield a slightly different result. As described above, any hypothetical gender or geographical patterns could not be formally explored due to the limited scope of the raw data. Furthermore, whereas the analysis was limited to comments and posts in English, there were also many YouTube viewer comments in Korean, Japanese, Chinese, and other languages. Due to the sheer volume of comments and the explorative nature of the study, it was not possible to have the non-English comments translated. Naturally, this limits the transferability of the findings to other language contexts, although it was obvious that many of the English comments included in the analysis were made by non-native English speakers from all over the world. Future research on the topic of mukbang should build on this explorative study and extend the analysis to include a broader scope of mukbang hosts, video material, and languages.

Another challenge for future research is to somehow negotiate access to the mukbang community in order to conduct formal interviews with viewers/participants as well as with mukbang hosts about their experiences within the scene. The netnographic approach used here is advantageous in research on sensitive topics where it may be difficult to negotiate access or recruit informants for a more 
traditional qualitative study. However, the approach naturally does not allow for an in-depth exploration with follow-up questions, informant clarifications, etc.

Furthermore, due to the inherently subjective nature of online user comments, we cannot know with any certainty that users that describe disordered eating actually suffer from a clinically relevant eating disorder. We do not wish to exaggerate any clinical implications of our findings; nevertheless, clinicians working with patients with eating disorders should be aware of the mukbang phenomenon as a potentially influencing factor.

\section{Conclusion}

In sum, this qualitative study adopting a netnographic approach shows that online users engage with the mukbang scene both as 'outsider' viewers and as 'insider' participants. The participant perspective includes descriptions about how watching mukbang may make some users limit or increase their own eating in a highly context-dependent manner. Not least, there is often a striking ambivalence in how users are influenced by and make sense of mukbang. For some, mukbang appears to be a constructive tool in increasing food intake, preventing binge eating, or reducing loneliness. For others, it is clearly a destructive force that may motivate restrictive eating or trigger a relapse into loss-of-control eating. Perhaps most notably, the mukbang phenomenon is not necessarily seen as either helpful or destructive, but instead as simultaneously useful and hurtful.

Acknowledgements Open access funding provided by Karolinska Institute.

Author Contributions MS conceived of and designed the study, collected and analyzed the study data, and drafted the manuscript. SAG supervised the study, analyzed the study data, and edited the manuscript. Both authors have approved the final manuscript.

Funding This research did not receive any specific grant from funding agencies in the public, commercial, or not-for-profit sectors.

\section{Compliance with Ethical Standards}

Conflict of interest The authors declare that they have no conflict of interest.

Open Access This article is licensed under a Creative Commons Attribution 4.0 International License, which permits use, sharing, adaptation, distribution and reproduction in any medium or format, as long as you give appropriate credit to the original author(s) and the source, provide a link to the Creative Commons licence, and indicate if changes were made. The images or other third party material in this article are included in the article's Creative Commons licence, unless indicated otherwise in a credit line to the material. If material is not included in the article's Creative Commons licence and your intended use is not permitted by statutory regulation or exceeds the permitted use, you will need to obtain permission directly from the copyright holder. To view a copy of this licence, visit http:// creativecommons.org/licenses/by/4.0/. 


\section{References}

Aladağ, Ahmet Emre, Serra Muderrisoglu, Naz Berfu Akbas, Oguzhan Zahmacioglu, and Haluk O. Bingol

2018 Detecting Suicidal Ideation on Forums: Proof-of-Concept Study. Journal of Medical Internet Research 20(6):e215.

Alexa Internet

2019 Traffic Statistics. Alexa Internet, Inc. Retrieved February 18, 2019. https://www.alexa.com/ siteinfo.

American Psychiatric Association

2013 DSM-5: Diagnostic and Statistical Manual of Mental Illness. 5th Edition. Washington, DC: American Psychiatry Publishing.

Bakhtin, Mikhail

1984 Rabelais and His World. Bloomington, IN: Indiana University Press.

Ballantine, Paul W., and Brett A.S. Martin

2005 Forming Parasocial Relationships in Online Communities. Advances in Consumer Research 32:197-201.

Baudrillard, Jean

1983 Simulations. Los Angeles, CA: Semiotext(e).

Chu, Marian

2018 How Bad are 'Mukbang' Shows, Really? Korea Biomedical Review. Retrieved February 27, 2019. https://www.koreabiomed.com/news/articleView.html?idxno=3890.

Debord, Guy

1992 Society of the Spectacle. London, UK: Rebel Press.

Derksen, Christina, Anna Serlachius, Keith J. Petrie, and Nicola Dalbeth

2017 "What Say ye Gout Experts?" A Content Analysis of Questions About Gout Posted on the Social News Website Reddit. BMC Musculoskeletal Disorders 18(1):488. https://doi.org/10.1186/ s12891-017-1856-y.

Diekema, David A.

1991 Televangelism and the Mediated Charismatic Relationship. The Social Science Journal 28 (2):143-162. https://doi.org/10.1016/0362-3319(91)90001-K.

Dyke, Sarah

2013 Utilising a Blended Ethnographic Approach to Explore the Online and Offline Lives of Pro-Ana Community Members. Ethnography and Education 8(2):146-161. https://doi.org/10.1080/ 17457823.2013.792505.

Fitzsimmons-Craft, Ellen E., Anna M. Bardone-Cone, Cynthia M. Bulik, Stephen A. Wonderlich, Ross D. Crosby, and Scott G. Engel

2014 Examining an Elaborated Sociocultural Model of Disordered Eating Among College Women: The Roles of Social Comparison and Body Surveillance. Body Image 11(4):488-500.

Google Trends

2018 Google Trends. Retrieved February 18, 2019. https://trends.google.com.

Gorse, Pauline, Clementine Nordon, Frederic Rouillon, Alexandra Pham-Scottez, and Anne Revah-Levy

2013 Subjective Motives for Requesting In-Patient Treatment in Female with Anorexia Nervosa: A Qualitative Study. PloS ONE 8(10):e77757.

Greenwood, Dara

2009 Idealized TV Friends and Young Women's Body Concerns. Body Image 6(2):97-104.

Hautala, Lea Anneli, Jouni Junnila, Hans Helenius, Aija-Mari Vaananen, Pirjo-Riitta Liuksila, Hannele Raiha, Maritta Valimaki, and Simo Saarijarvi

2008 Towards Understanding Gender Differences in Disordered Eating Among Adolescents. Journal of Clinical Nursing 17(13):1803-1813.

Hoffner, Cynthia

1996 Children's Wishful Identification and Parasocial Interaction with Favorite Television Characters. Journal of Broadcasting \& Electronic Media 40(3):389-402. https://doi.org/10.1080/ 08838159609364360.

Horton, Donald, and Richard R. Wahl

1956 Mass Communication and Para-Social Interaction: Observations on Intimacy at a Distance. Psychiatry 19:215-229. 
Hsieh, Hsiu-Fang, and Sarah E. Shannon

2005 Three Approaches to Qualitative Content Analysis. Qualitative Health Research 15(9):12771288.

Jackson, Katie

2018 Inside "Mukbang": How Some Professional Binge-Eaters Earn Thousands. Today FOOD. Retrieved February 27, 2019. https://www.today.com/food/what-mukbang-inside-viral-koreanfood-phenomenon-t123251.

Jackson, Safia C., Pamela K. Keel, and Young Ho Lee

2006 Trans-Cultural Comparison of Disordered Eating in Korean Women. International Journal of Eating Disorders 39(6):498-502. https://doi.org/10.1002/eat.20270.

Jeon, Yongwoog Andrew, Brent Hale, Eric Knackmuhs, and Michael Mackert

2018 Weight Stigma Goes Viral on the Internet: Systematic Assessment of YouTube Comments Attacking Overweight Men and Women. Interactive Journal of Medical Research 7(1):e6.

Johnson, Adrienne Rose

2011 The Slim Eater. Competitive Eating and American Culture. Appetite 56(2):533.

Jung, Jaehee, and Gordon B. Forbes

2007 Body Dissatisfaction and Disordered Eating among College Women in China, South Korea, and the United States: Contrasting Predictions from Sociocultural and Feminist Theories. Psychology of Women Quarterly 31(4):381-393. https://doi.org/10.1111/j.1471-6402.2007.00387.x.

Kim, Jihyun, and Hayeon Song

2016 Celebrity's Self-Disclosure on Twitter and Parasocial Relationships: A Mediating Role of Social Presence. Computers in Human Behavior 62:570-577.

Kim, Mingu

2017 Extreme Eating: Are "Mukbangs" Helpful or Hurtful to Those With Eating Disorders? Students in Mental Health Research Blog. Retrieved February 18, 2019. https://www.hcs.harvard.edu/hcht/ blog/eating-disorder.

Kozinets, Robert V.

1998 On Netnography: Initial Reflections on Consumer Research Investigations of Cyberculture. Advances in Consumer Research 25:366-371.

Langer, Roy, and Suzanne C. Beckman

2005 Sensitive Research Topics: Netnography Revisited. Qualitative Market Research: An International Journal 8(2):189-203.

Lavis, Anna

2015 Food Porn, Pro-Anorexia and the Viscerality of Virtual Affect: Exploring Eating in Cyberspace. Geoforum 84:198-205.

Lester, Rebecca

2014 Health as Moral Failing: Medication Restriction Among Women with Eating Disorders. Anthropology \& Medicine 21(2):241-250.

Lewis, Stephen P., Nancy L. Heath, Michael J. Sornberger, and Alexis E. Arbuthnott

2012 Helpful or Harmful? An Examination of Viewers' Responses to Nonsuicidal Self-Injury Videos on YouTube. Journal of Adolescent Health 51(4):380-385.

McLuhan, Marshall

1964 Understanding Media: The Extension of Man. New York City, NY: McGraw-Hill.

Musolino, Connie, Megan Warin, Tracey Wade, and Peter Gilchrist

2015 'Healthy Anorexia': The Complexity of Care in Disordered Eating. Social Science \& Medicine 139:18-25.

O'Neil-Hart, Celie, and Howard Blumenstein

2016 Why YouTube Stars are More Influential than Traditional Celebrities. Think with Google. Retrieved December 17, 2019. https://www.thinkwithgoogle.com/consumer-insights/youtubestars-influence.

Oksanen, Atte, David Garcia, Anu Sirola, Matti Näsi, Markus Kaakinen, Teo Keipi, and Pekka Räsänen

2015 Pro-Anorexia and Anti-Pro-Anorexia Videos on YouTube: Sentiment Analysis of User Responses. Journal of Medical Internet Research 17(11):e256.

Pike, Kathleen M., and Patricia E. Dunne

2015 The Rise of Eating Disorders in Asia: A Review. Journal of Eating Disorders 3:33.

Pila, Eva, Jonathan M. Mond, Scott Griffiths, Deborah Mitchison, and Stuart B. Murray

2017 A Thematic Content Analysis of \#Cheatmeal Images on Social Media: Characterizing an Emerging Dietary Trend. International Journal of Eating Disorders 50(6):698-706. 
Poerio, Giulia Lara, Emma Blakey, Thomas J. Hostler, and Theresa Veltri

2018 More than a Feeling: Autonomous Sensory Meridian Response (ASMR) is Characterized by Reliable Changes in Affect and Physiology. PLoS ONE 13(6):1-18. https://doi.org/10.1371/ journal.pone.0196645.

Quartz

2016 Why Some Koreans Make $\$ 10,000$ a Month to Eat on Camera. Quartz. Retrieved February 18, 2019. https://qz.com/592710/why-some-koreans-make-10000-a-month-to-eat-on-camera.

Russo, Mary

1994 The Female Grotesque: Risk, Excess and Modernity. London, UK: Routledge.

Shohet, Merav

2007 Narrating Anorexia: "Full" and "Struggling" Genres of Recovery. Ethos 35(3):344-382.

Shohet, Merav

2017 Beyond the Clinic? Eluding a Medical Diagnosis of Anorexia Through Narrative. Transcultural Psychiatry 55(4):495-515. https://doi.org/10.1177/1363461517722467.

Sowles, Shaina J., Melissa J. Krauss, Lewam Gebremedhn, and Patricia A. Cavazos-Rehg

2017 "I Feel like I've Hit the Bottom and have no Idea What to Do": Supportive Social Networking on Reddit for Individuals with a Desire to Quit Cannabis Use. Substance Abuse 38(4):477-482. https://doi.org/10.1080/08897077.2017.1354956.

Sowles, Shaina J., Monique McLeary, Allison Optican, Elizabeth Cahn, Melissa J. Krauss, Ellen E.

Fitzsimmons-Craft, Denise E. Wilfley, and Patricia A. Cavazos-Rehg

2018 A Content Analysis of an Online Pro-Eating Disorder Community on Reddit. Body Image 24:137-144.

Tan, Jacinta O.A., Tony Hope, Anne Stewart, and Raymond Fitzpatrick

2003 Control and Compulsory Treatment in Anorexia Nervosa: the Views of Patients and Parents. International Journal of Law and Psychiatry 26(6):627-645.

Tenderich, Amy, Burghardt Tenderich, Tanner Barton, and Sarah Elizabeth Richards

2019 What Are PWDs (People With Diabetes) Doing Online? A Netnographic Analysis. Journal of Diabetes Science and Technology 13(2):187-197. https://doi.org/10.1177/1932296818813192.

Veblen, Thorstein

2009 The Theory of the Leisure Class. Oxford, UK: Oxford University Press.

Warin, Megan

2004 Primitivising Anorexia: The Irresistible Spectacle of Not Eating. Australian Journal of Anthropology 15(1):95-104. https://doi.org/10.1111/j.1835-9310.2004.tb00368.x.

Warin, Megan

2010 Abject Relations: Everyday Worlds of Anorexia. Piscataway, NJ: Rutgers University Press.

Young, Ariana F, Shira Gabriel, and Jordan L Hollar

2013 Batman to the Rescue! The Protective Effects of Parasocial Relationships with Muscular Superheroes on Men's Body Image. Journal of Experimental Social Psychology 49(1):173-177.

Publisher's Note Springer Nature remains neutral with regard to jurisdictional claims in published maps and institutional affiliations. 